Research Paper

\title{
Novel combination of celecoxib and metformin improves the antitumor effect by inhibiting the growth of Hepatocellular Carcinoma
}

\author{
Jun-Wen $\mathrm{Hu}^{*}$, Bin Chen*, Jie Zhang, Ya-Peng Qi, Jia-Hao Liang, Jian-Hong Zhong ${ }^{\bowtie}$ and Bang-De Xiang ${ }^{\bowtie}$ \\ Hepatobiliary Surgery Department, Guangxi Liver Cancer Diagnosis and Treatment Engineering and Technology Research Center, Key Laboratory for \\ High-Incidence Tumor Prevention and Treatment, Ministry of Education, Guangxi Medical University Cancer Hospital, Nanning, China. \\ *These authors contributed equally to this work. \\ $\triangle$ Corresponding authors: Bang-De Xiang and Jian-Hong Zhong, Hepatobiliary Surgery Department, Guangxi Medical University Cancer Hospital, \#71, He Di \\ Rd. Nanning 530021, China. Phone: +86-0771-5330855 (office). Fax: +86-0771-5312000. E-mail: xiangbangde@gxmu.edu.cn (B.-D.X); zhongjianhong@gxmu.edu.cn \\ (J-H.Z). \\ ( ) The author(s). This is an open access article distributed under the terms of the Creative Commons Attribution License (https://creativecommons.org/licenses/by/4.0/). \\ See http://ivyspring.com/terms for full terms and conditions.
}

Received: 2020.04.28; Accepted: 2020.08.27; Published: 2020.09.14

\begin{abstract}
Objective: To explore the effect of COX-2 inhibitor celecoxib in combination with metformin on the prevention of Hepatocellular carcinoma (HCC) and the mechanisms involved.

Methods: HCC cell lines and an HCC rat model were treated with celecoxib, metformin or a combination of both. Cell viability and tumor formation were measured.

Results: In vitro and in vivo studies showed that treatment with a combination of celecoxib and metformin inhibited proliferation of HCC to a greater extent than either treatment alone, by reducing the phosphorylation of MTOR.

Conclusion: The study suggested that celecoxib combined with metformin would be more effective for the preventing occurrence of HCC than either treatment alone and this combination of therapy is worthy of further study.
\end{abstract}

Key words: Celecoxib, metformin, Hepatocellular carcinoma, combination, MTOR

\section{Introduction}

Hepatocellular carcinoma (HCC) is the most destructive and invasive form of liver cancers. The incidence of HCC continues to rise rapidly, ranking with the sixth most common cancer and the fourth leading cause of cancer death worldwide [1]. Although the clinical diagnosis and treatment of early HCC have improved significantly, the prognosis of HCC is still very poor [2,3]. In addition, highly invasive and advanced HCC responds minimally or not at all to general treatment [4-6]. Therefore, there is an urgent need for new valid and well-tolerated treatment strategies. Targeted therapy has entered the field of anti-tumor therapy, bringing hope for the treatment of HCC [7-9]. However, the current targeted therapy drugs generally have low tumor response rates and substantial side effects, so it is necessary to explore other types of targeted therapy against HCC.

HCC is usually the result of continuous damage and chronic inflammation. An important inflammatory mediator is the inducible gene cyclooxygenase-2 (COX-2) [10-13]. COX-2 is widely expressed in various types of cancer, including liver cancer, and it promotes tumor progression and cancer cells' resistance to chemotherapy and radiation therapy [14]. COX-2 is now well established as an important molecular target for anti-cancer therapy. COX-2 inhibitors have demonstrated potential therapeutic effects in HCC [15-17]. Celecoxib is the COX-2 selective inhibitor which may help slow the progression of lung, breast, liver, colon and prostate tumors [18-22]. 
Metformin (MET) is a first-line anti-diabetic drug for the treatment of type 2 diabetes [23,24]. More importantly, the anticancer effect of MET has been widely reported in recent years [25-27]. In addition, MET protects the liver from chemicals or viral hepatotoxicants [28]. Interestingly, it has been reported that the combined use of MET and aspirin can inhibit the growth and metastatic potential of liver cancer in vitro [29].

Molecular targeted therapy does bring promise for HCC, however, as in most cancers, the use of single molecular targeted drugs is unlikely to achieve long-term relief or cure in HCC, especially for advanced stages of disease [7,30]. Therefore, combination therapies will be necessary, the combination of two or more anticancer drugs that target cancer in different ways has been considered a promising treatment strategy that maximizes the efficacy of the drug and reduces the side effects associated with a single component to lowest, so there seems to be reason to speculate that a combination of drugs will ultimately increase treatment benefits.

In this study, we aim to investigate the enhanced effect of Celecoxib band MET combination treatment on tumorigenesis in vitro and in vivo.

\section{Materials and Methods}

\section{Reagents and drugs}

The rabbit monoclonal antibody against phosphorylated mTOR (p-mTOR) (Ser2448) (49F9) was purchased from CST Corporation (USA). Celecoxib capsules and metformin hydrochloride capsules were provided by Guangxi Medical University Cancer Hospital (Nanning, China).

\section{Cell lines}

Human HCC cell lines HepG2 and HCCLM3 were purchased from the Cell Resource Center of Shanghai Institutes for Biological Sciences (Chinese Academy of Sciences, China). The cells were cultured in a complete medium (89\% DMEM $+10 \%$ fetal bovine serum $+1 \%$ penicillin and streptomycin) at $37^{\circ} \mathrm{C}$ in a humidified atmosphere with $5 \% \mathrm{CO}_{2}$.

\section{MTT assay for cell viability}

Cell viability was determined using the MTT assay (Sigma, USA) according to the manufacturer's instructions. HepG2 and HCCLM3 cells were seeded at a density of $10^{4}$ cells per well in 96-well plates for 24 $\mathrm{h}$. Then the cells were treated for $48 \mathrm{~h}$ with $50 \mu \mathrm{M}$ celecoxib, $500 \mu \mathrm{M}$ of metformin, or the combination of $50 \mu \mathrm{M}$ celecoxib and $500 \mu \mathrm{M}$ metformin; control cells were treated with an equal volume of phosphate buffered saline (PBS). After treatment, $20 \mu \mathrm{L}$ of MTT solution $(5 \mathrm{mg} / \mathrm{ml})$ was added to each well and the plates were incubated at $37{ }^{\circ} \mathrm{C}$ for $4 \mathrm{~h}$. Cells were lysed by adding DMSO and the optical density of each well was measured at $450 \mathrm{~nm}$. Viability was calculated as a percentage of control cells.

\section{Animals}

A total of 98 healthy, male Sprague-Dawley (SD) rats (6 weeks old) weighing 150-200 g were provided by the Animal Experiment Center of Guangxi Medical University. Animals were maintained in a specific pathogen-free (SPF) room under laminar flow and given sterilized food and water.

\section{In vivo tumorigenesis experiment}

The rats were divided into 5 groups, 10 rats were randomly selected as the normal control group, without special treatment during the experiment, all the remaining 88 rats were intraperitoneally injected with DEN dissolved with saline solution $(50 \mathrm{mg} / \mathrm{kg}$, once / week for 18 weeks), then they were randomly divided into four group ( $\mathrm{n}=22$ in each group) as follows: placebo group, metformin-treated (300 $\mathrm{mg} / \mathrm{kg})$ group, celecoxib-treated $(100 \mathrm{mg} / \mathrm{kg})$ group, and combination-treated (metformin + celecoxib) group. Drug treatment was administered daily for 18 weeks by oral gavage and the placebo group received an equal volume of saline solution by oral gavage. The dose of metformin and celecoxib was chosen according to previous preclinical researches and is anticipated to be innoxious. Throughout the in vivo experiment, normal diet was maintained for all rat groups. Body weight was measured once a week and the general behavior of the rats, such as their activity, mentality, eating, feces and hair color, were closely observed during the experiment. At the end of week 19 , all the rats were sacrificed by intraperitoneal injection of $10 \%$ chloral hydrate. Liver tissues from each rat were fixed in formaldehyde for hematoxylineosin (HE) and immunohistochemical staining.

\section{Immunohistochemical staining}

Paraffin sections were baked in an oven at $65^{\circ} \mathrm{C}$ for $2 \mathrm{~h}$. After dewaxing with xylene and dehydrating with gradient alcohol, antigen retrieval was performed by immersing sections in citric acid solution at $100{ }^{\circ} \mathrm{C}$ for $15 \mathrm{~min}$. Then sections were cooled to room temperature and washed twice with PBS. Sections were treated with 3\% hydrogen peroxide and incubated at $37{ }^{\circ} \mathrm{C}$ for 10 minutes, washed 3 times with PBS, and blocked with normal goat serum. The sections were then incubated overnight at $4{ }^{\circ} \mathrm{C}$ with p-mTOR antibody (working concentration 1: 100). Tissue slices were washed with PBS three times, incubated with biotinylated secondary antibody (Zhongshan Golden Bridge Biotechnology Company, Beijing China) for $30 \mathrm{~min}$ at 
room temperature. After another PBS wash, sections were incubated for $30 \mathrm{~min}$ with streptavidinperoxidase complex (Zhongshan Golden Bridge Biotechnology Company, Beijing China). The color reaction was developed with diaminobenzidine, finally, sections were counterstained with hematoxylin. Tumors with $>25 \%$ p-mTOR positive cells were deemed positive for antigen expression. Pathological diagnoses of tissue sections were determined by two experienced pathologists blinded to treatment.

\section{Statistical analyses}

All data during the experiment were analyzed by SPSS22.0 software. Quantitative data are described by means \pm standard deviation. The comparison of the means between groups was performed by one-way analysis of variance, followed by the least significant difference (LSD) method; the statistics of the composition ratio or categorical variable rate were tested using the chi-squared test or Fisher's exact probability method. Differences associated with $P$ $<0.05$ were considered statistically significant.

\section{Results}

\section{Celecoxib and metformin combination treatment significantly reduces $\mathrm{HCC}$ cell survival in vitro}

The combination treatment of celecoxib and metformin displayed significantly higher cytotoxicity than either drug alone (Figure 1, Table 1). Cell survival was similar after celecoxib or metformin treatment alone.

Table 1. Cell survival of drug-treated hepatocellular carcinoma cell lines, as a percentage of survival in control cells

\begin{tabular}{llll}
\hline Cell line & $50 \mu \mathrm{M} \mathrm{Cel}$ & $500 \mu \mathrm{M}$ Met & $50 \mu \mathrm{M} \mathrm{Cel}+500 \mu \mathrm{M}$ Met \\
\hline HepG2 & $81.43 \pm 3.12$ & $78.07 \pm 2.54$ & $39.12 \pm 3.16^{\mathrm{a}}$ \\
HCCLM3 & $70.59 \pm 5.95$ & $64.65 \pm 4.21$ & $49.97 \pm 5.43^{\mathrm{b}, \mathrm{c}}$ \\
\hline
\end{tabular}

Values are presented as mean $\pm \mathrm{SD}$;

a $P<0.001$ compared to celecoxib or metformin treatment alone;

b $P<0.01$ compared to celecoxib treatment alone;

c $P<0.05$ compared to metformin treatment alone;

Cel, celecoxib; Met, metformin.

\section{Combination treatment significantly reduces HCC tumor formation in rats}

The rats in the normal control group gained significantly more weight over time than the treatment groups (Figure 2A). Notably, the rats treated with a combination of drugs gained more weight than other experimental rats beginning at week 12; however, the differences failed to reach statistical significance. Before rats were sacrificed at week 19, a small number of rats in each experimental group died prematurely due to intestinal obstruction, gastrointestinal perforation, and intestinal necrosis caused by side effects.

Based on gross anatomy and HE staining, the final tumor formation rate in each group was as follows: placebo group, $65.0 \%$; celecoxib group, $36.8 \%$; metformin group, $42.9 \%$; and combination drug group, $17.6 \%$ (Figure 2B, Table 2). Combination treatment led to significantly lower tumor formation rate than placebo.

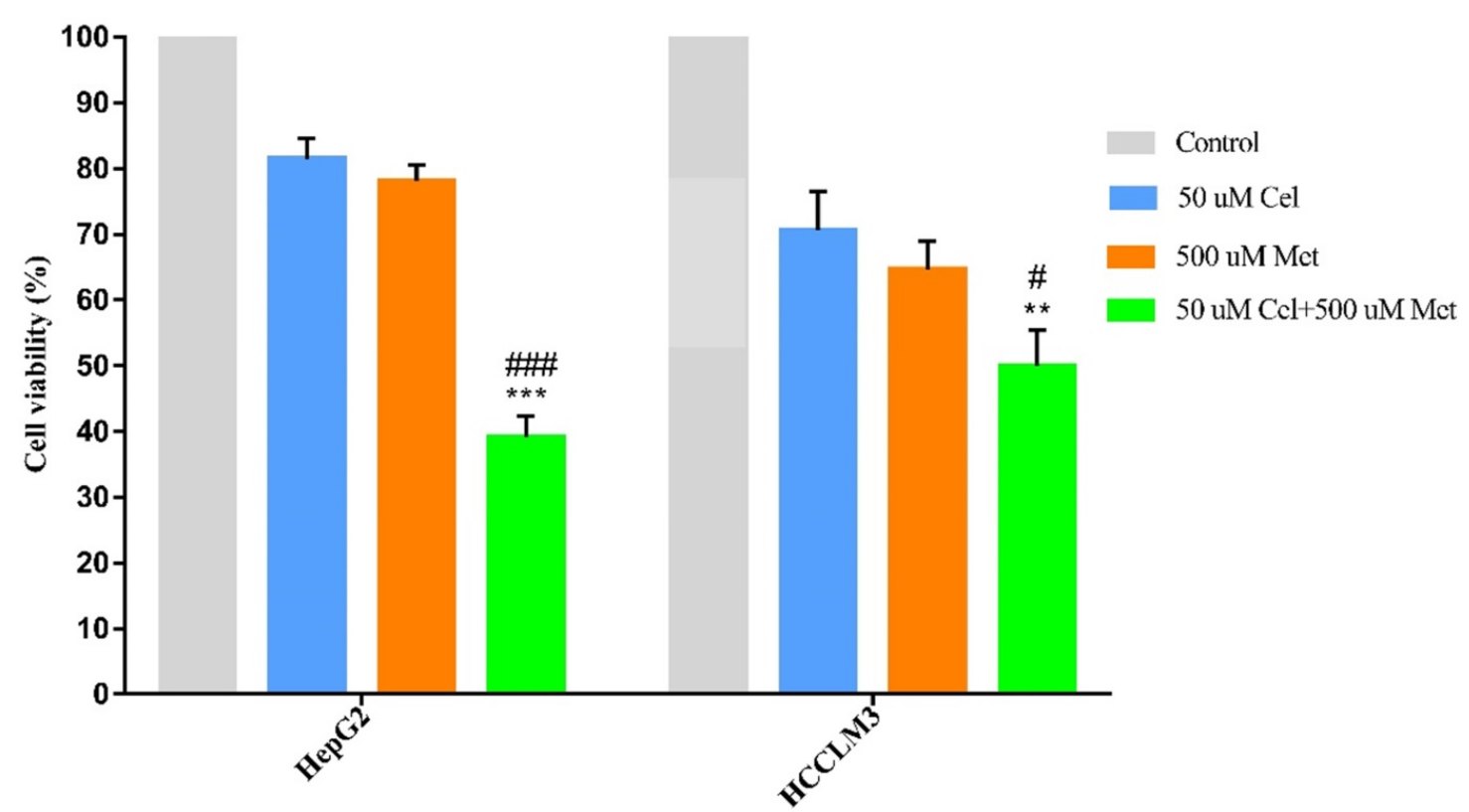

Figure 1. Effect of celecoxib, metformin and their combination on cell survival in HCC cell lines. HepG2 and HCCLM3 cells were treated with the specified dosage of celecoxib and metformin either alone or in combination for $48 \mathrm{~h}$. Then MTT assay was used to measure cell viability. Data are represented as a percent of control cells and are the mean \pm SD of three independent experiments. $* * P<0.01$, $* * * P<0.001$ compared with celecoxib alone; $\# P<0.05$, \#\#P<0.001 compared with metformin alone. 

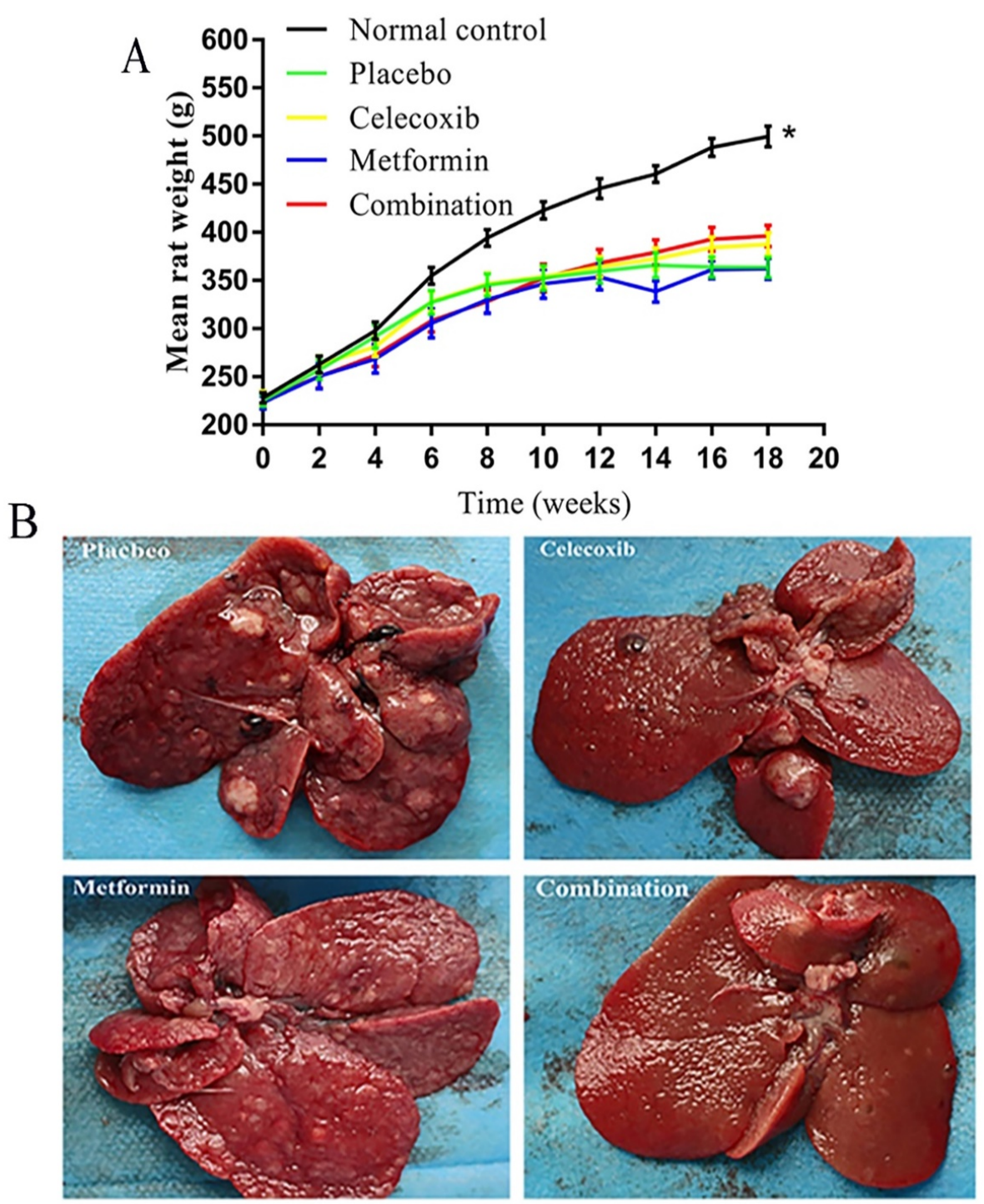

Figure 2. Effects of celecoxib and metformin alone and in combination on the growth of hepatocellular carcinoma in rats. (A) Quantification of rat weights in each group measured over time. $* P<0.05$ versus control. (B) Photographs of tumors in representative livers isolated from rats in each treatment group at the end of the 18 weeks of treatment.

Table 2. Comparison of hepatocellular carcinoma tumor formation in rats after drug treatment

\begin{tabular}{lllll}
\hline Oncogenesis & $\begin{array}{l}\text { placebo } \\
(\mathrm{n}=20)\end{array}$ & $\begin{array}{l}\text { celecoxib } \\
(\mathrm{n}=19)\end{array}$ & $\begin{array}{l}\text { metformin } \\
(\mathrm{n}=14)\end{array}$ & $\begin{array}{l}\text { combination } \\
(\mathrm{n}=17)\end{array}$ \\
\hline No & $7(35)$ & $12(63.2)$ & $8(57.1)$ & $14(82.4)^{\mathrm{a}}$ \\
Yes & $13(65)$ & $7(36.8)$ & $6(42.9)$ & $3(17.6)$ \\
\hline
\end{tabular}

Values are shown as $\mathrm{n}(\%)$;

a $P<0.05$ compared to the placebo group.

\section{Combination treatment reduces $\mathrm{p}-\mathrm{mTOR}$ in cancerous liver tissue}

Immunohistochemistry showed that p-mTOR was highly increased in liver tissue from all rats with HCC. The group given combination treatment showed the lowest positivity of p-mTOR among all groups. However, positive rates between the groups were not statistically significant, which may be due to the small number of samples (Figure 3, Table 3).

Table 3. Comparison of $\mathrm{p}-\mathrm{mTOR}$ positivity in liver sections from rats with induced hepatocellular carcinoma after drug treatment

\begin{tabular}{lllll}
\hline Positivity & $\begin{array}{l}\text { placebo } \\
(\mathrm{n}=18)\end{array}$ & $\begin{array}{l}\text { celecoxib } \\
(\mathrm{n}=15)\end{array}$ & $\begin{array}{l}\text { metformin } \\
(\mathrm{n}=12)\end{array}$ & $\begin{array}{l}\text { combination } \\
(\mathrm{n}=14)\end{array}$ \\
\hline No & $7(38.9 \%)$ & $7(46.7 \%)$ & $7(58.3 \%)$ & $9(64.3 \%)$ \\
Yes & $11(61.1 \%)$ & $8(53.3 \%)$ & $5(41.7 \%)$ & $5(35.7 \%)$ \\
\hline \multicolumn{7}{l}{ Values are expressed as $\mathrm{n}(\%)}$. & & &
\end{tabular}

\section{Discussion}

HCC is an extremely complex tumor that requires effective treatment through multi-pronged approach. Finding more effective drugs to treat HCC is of great significance. Monotherapy for cancer may produce chemotherapy resistance, whereas a great number of researches have revealed that combined 
treatment can enhance antitumor effects [31-34]. The multi-target-based approach appears more appropriate for treating HCC, implying that combination therapy should be more effective.

Celecoxib is a first-line non-steroidal antiinflammatory drug and a potent inhibitor of COX-2. It has shown anti-cancer activity in many different types of cancer cells and animal models including liver cancer [35-40]. Many previous studies have found that celecoxib can not only inhibit the proliferation of a variety of tumors, but also induce apoptosis of tumor cells. Moreover its inhibition of cancer cells appears to be time and concentration dependent [41-43]. Celecoxib is effective against human epithelial cell type tumors, the US Food and Drug Administration (FDA) has approved oral celecoxib for patients with familial colon adenoma polyps to prevent colon cancer [44].

Metformin is a widely used in the clinic as an oral hypoglycemic drug. Recently, the therapeutic effect of metformin on tumors has attracted attention. Many studies have shown that metformin can also treat tumors, such as those found in lung, breast, colon and prostate [45-47]. Metformin may reduce the risk of $\mathrm{HCC}$ in patients with diabetes, implying that metformin may also have a therapeutic effect against HCC [48]. The proposed mechanism underlying this therapeutic effect is that metformin inhibits cell growth and promotes apoptosis in a dose-dependent manner [49].

Previous studies have determined the effects of either metformin or celecoxib together with standard chemotherapeutics. When metformin is combined with gefitinib, it can produce stronger cytotoxicity in lung squamous cancer cells, and enhance the growth inhibition of lung cancer cells [50]. Metformin can enhance the sensitivity of ovarian cancer cell lines to carboplatin when given in combination [51]. Together with ionizing radiation, metformin enhances cytotoxicity and inhibits DNA repair in liver cancer cells [52]. Similarly, celecoxib in combination with Bacillus Calmette-Guerin immunotherapy is more effective against urothelial cell carcinoma than intravesical therapy alone [53]. Celecoxib combined with fluvastatin is more effective against HCC than either treatment alone [54]. The combination of celecoxib with the multikinase inhibitor sorafenib produces a synergistic apoptotic effect against liver cancer cells [55]. These studies have shown that combination medication is better than single medication in treating many types of cancer cells.

A previous study investigating the combined application of metformin and celecoxib found that together they synergistically inhibited cell proliferation in a concentration-dependent manner. Since this increase in cytotoxicity does not increase DNA damage, this combination can be used to inhibit the growth of malignant cells without any genotoxic or mutational effects at the cellular level [56]. Therefore, combined molecular targeted therapy can be used for certain types of advanced malignancies. Metformin combined with celecoxib may be a viable option to treat or prevent tumors, especially in obese and diabetic patients.

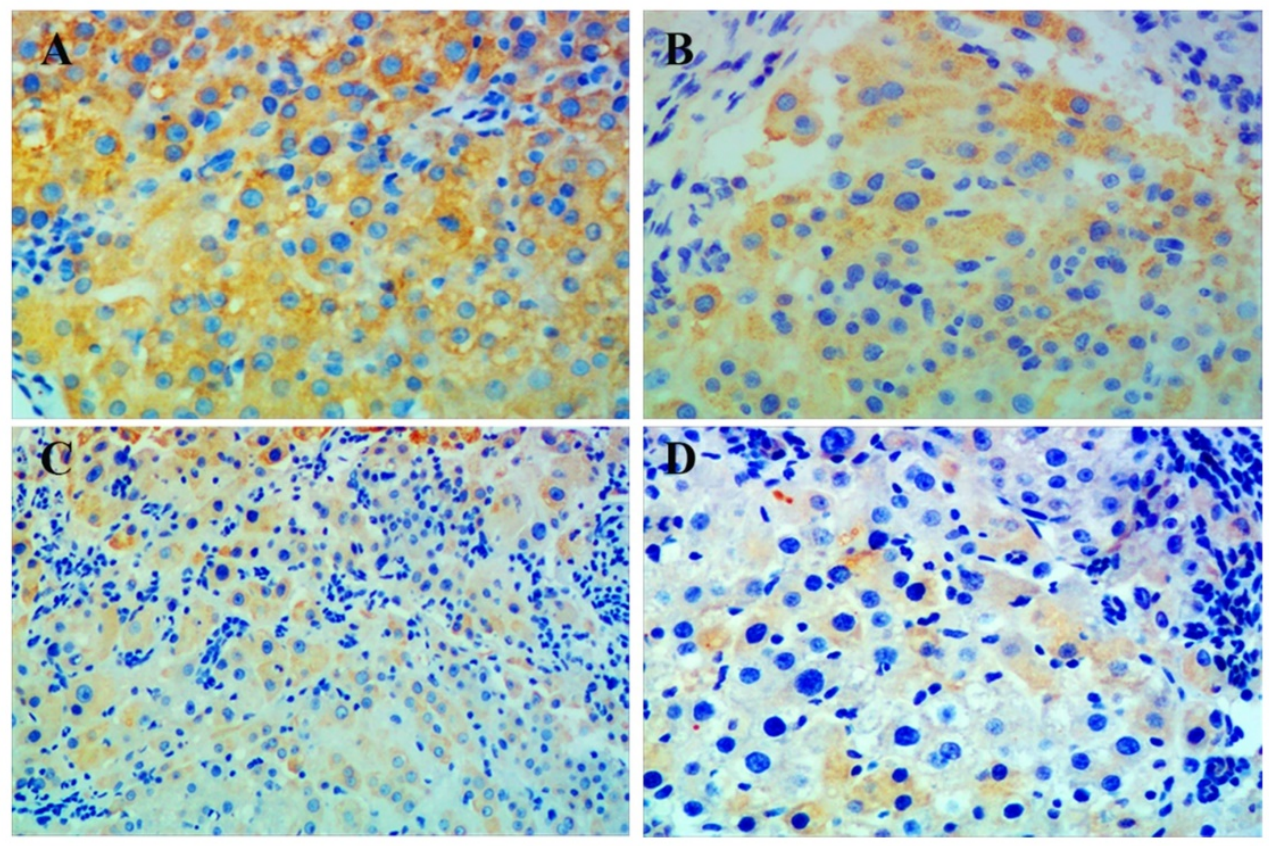

Figure 3. Immunohistochemical staining for $\mathrm{p}$-mTOR in liver tissue. Representative images of liver tissue isolated from rats with hepatocellular carcinoma and treated with (A) placebo, (B) celecoxib, (C) metformin, or (D) combined metformin and celecoxib. Magnification $\times 200$. 
In order to verify whether the combined use of metformin and celecoxib can effectively prevent the occurrence of HCC, we first tested the combined effects in vitro by MTT assay. Then we established an HCC rat model to explore the antitumor effects. The results showed that treatment with celecoxib or metformin alone only produced negligible decreases in tumor formation rate. In contrast, combined medication significantly reduce tumor formation rate in rats. Our research shows that the combination of drugs is more effective than either drug alone in inhibiting the occurrence of liver cancer.

mTOR is a key downstream gene in many signaling pathways, and its increased phosphorylation level can promote tumor cell growth and development, conversely reducing the phosphorylation level of mTOR can inhibit these pathways and then restrain the tumorigenesis and development of tumors [57,58]. Celecoxib or metformin in combination with other drugs reduces the phosphorylation level of mTOR by inactivating the $\mathrm{PI} 3 \mathrm{~K} / \mathrm{Akt} / \mathrm{mTOR}$ or AMPK/mTOR signaling pathways, resulting in tumor inhibition [59-61].

In order to further study the anti-hepatic mechanism of celecoxib combined with metformin against HCC, we used immunohistochemical staining to detect p-mTOR in liver tissue of each group of rats, and found that the positive expression rate of p-mTOR in the combination group was lowest. Our results suggested that celecoxib combined with metformin may synergistically suppress mTORrelated signaling pathways by reducing mTOR phosphorylation, thereby inhibiting tumorigenesis.

We have confirmed for the first time in vitro and vivo experiments that celecoxib combined with metformin can synergistically inhibit the occurrence of liver cancer, and we have initially revealed the mechanism. However, our findings have some limitations. First, the number of our experimental samples was relatively small. A small proportion of rats in the experimental groups died from intestinal obstruction, gastrointestinal perforation, and intestinal necrosis due to drug side effects. Second, we studied only one mechanism that may underlie the therapeutic efficacy of the combined therapy. Given the complexity of molecular pathways in cancer development and pharmaceutical regulation, further studies should explore other potential mechanisms.

In summary, our results suggest that celecoxib combined with metformin may inhibit the occurrence and development of liver cancer, as demonstrated in rat models of HCC and appears to involve synergistic inhibition of mTOR-related signaling pathways. Larger studies are required to assess and maximize the safety and efficacy of celecoxib and metformin in the treatment of HCC, and to explore the molecular mechanism of synergistic inhibition of tumors before clinical application.

\section{Abbreviations}

HCC, Hepatocellular carcinoma; COX-2, cyclooxygenase-2; MET, Metformin; DEN, diethylnitrosamine; SPF, specific pathogen free; HE, hematoxylin-eosin; PBS, phosphate buffered saline; OD, Optical density; BCG, Bacillus Calmette-Guerin.

\section{Acknowledgments}

\section{Author biography}

Dr. Bandde Xiang is the leader of the Department of Hepatobiliary Surgery of Guangxi Medical University Cancer Hospital, the deputy director of the Department of Surgery of the Oncology School of Guangxi Medical University, and a doctoral tutor. He has profound knowledge in basic research and clinical work of primary liver cancer, has a solid clinical foundation and rich scientific research experience, is proficient in cell biology, molecular biology and animal experiment technology, and has been published more than 30 articles related to basic and clinical research of liver cancer as the first or corresponding author, and has undertaken and completed a number of research projects related to liver cancer. Among them, "the establishment and application of the system for prevention and treatment of postoperative recurrence of primary liver cancer" won the first prize of Guangxi Science and Technology Progress Award in 2017, "the expansion and clinical application of liver cancer surgical indications" won the first prize of Guangxi Science and Technology Progress Award in 2019.

\section{Funding}

National Natural Science Foundation of China (81960450), the National Major Special Science and Technology Project(2017ZX10203207), High-level innovation team and outstanding scholar program in Guangxi Colleges and Universities, "139" projects for training of high-level medical science talents from Guangxi, The Key Research and Development Project of Guangxi (AA18221001, AB18050020), the Key laboratory of High-Incidence-Tumor Prevention \& Treatment (Guangxi Medical University), Ministry of Education (GKE2017-ZZ02, GKE2018-KF02 and GKE2019-07), Development and application of medical and health appropriate technology in Guangxi (S2019039) and 'Guangxi Ba Gui Scholars' Special Fund. 


\section{Competing Interests}

The authors have declared that no competing interest exists.

\section{References}

1. Bray F, Ferlay J, Soerjomataram I, et al. Global cancer statistics 2018: GLOBOCAN estimates of incidence and mortality worldwide for 36 cancers in 185 countries. CA Cancer J Clin 2018; 68: 394-424.

2. Balogh J, Victor DR, Asham EH, et al. Hepatocellular carcinoma: a review. J Hepatocell Carcinoma 2016; 3: 41-53.

3. Llovet JM, Zucman-Rossi J, Pikarsky E, et al. Hepatocellular carcinoma. Nat Rev Dis Primers 2016; 2: 16018.

4. Chen LT, Martinelli E, Cheng AL, et al. Pan-Asian adapted ESMO Clinical Practice Guidelines for the management of patients with intermediate and advanced/relapsed hepatocellular carcinoma: a TOS-ESMO initiative endorsed by CSCO, ISMPO, JSMO, KSMO, MOS and SSO. Ann Oncol 2020; 31: 334-51.

5. Vogel A and Saborowski A. Current strategies for the treatment of intermediate and advanced hepatocellular carcinoma. Cancer Treat Rev 2020; 82: 101946.

6. Bouattour M, Mehta N, He AR, et al. Systemic Treatment for Advanced Hepatocellular Carcinoma. Liver cancer 2019; 8: 341-58.

7. Dimri $M$ and Satyanarayana A. Molecular Signaling Pathways and Therapeutic Targets in Hepatocellular Carcinoma. Cancers 2020;12(2):491.

8. Chen S, Cao Q, Wen W, et al. Targeted therapy for hepatocellular carcinoma: Challenges and opportunities. Cancer Letters 2019; 460: 1-9.

9. Greten TF, Lai CW, Li G, et al. Targeted and Immune-Based Therapies for Hepatocellular Carcinoma. Gastroenterology 2019; 156: 510-24.

10. Kwon SH, Jeong SW, Jang JY et al. Cyclooxygenase-2 and vascular endothelial growth factor in chronic hepatitis, cirrhosis and hepatocellular carcinoma. Clin Mol Hepatol 2012; 18: 287-94

11. Giannitrapani L, Ingrao S, Soresi $\mathrm{M}$ et al. Cyclooxygenase-2 expression in chronic liver diseases and hepatocellular carcinoma: an immunohistochemical study. Ann N Y Acad Sci 2009; 1155: 293-99.

12. Cusimano A, Fodera D, Lampiasi $\mathrm{N}$ et al. Prostaglandin $\mathrm{E} 2$ receptors and COX enzymes in human hepatocellular carcinoma: role in the regulation of cell growth. Ann N Y Acad Sci 2009; 1155: 300-8.

13. Cervello M, Foderaa D, Florena AM et al. Correlation between expression of cyclooxygenase- 2 and the presence of inflammatory cells in human primary hepatocellular carcinoma: possible role in tumor promotion and angiogenesis. World J Gastroenterol 2005; 11: 4638-43.

14. Hashemi Goradel N, Najafi M, Salehi E, et al. Cyclooxygenase-2 in cancer: A review. J Cell Physiol 2019; 234: 5683-99.

15. Lampiasi N, Azzolina A, Umezawa $\mathrm{K}$ et al. The novel NF- $\kappa \mathrm{B}$ inhibitor DHMEQ synergizes with celecoxib to exert antitumor effects on human liver cancer cells by a ROS-dependent mechanism. Cancer Letters 2012; 322: 35-44.

16. Cusimano A, Fodera D, Dalessandro N, et al. Potentiation of the antitumor effects of both selective cyclooxygenase- 1 and cyclooxygenase- 2 inhibitors in human hepatic cancer cells by inhibition of the MEK/ERK pathway. Cancer Biol Ther 2007; 6: 1461-68.

17. Lampiasi N, Fodera D, Dalessandro N, et al. The selective cyclooxygenase-1 inhibitor SC-560 suppresses cell proliferation and induces apoptosis in human hepatocellular carcinoma cells. Int J Mol Med 2006; 17: 245-52.

18. Qiu Z, Zhang C, Zhou J et al. Celecoxib alleviates AKT/c-Met-triggered rapid hepatocarcinogenesis by suppressing a novel COX-2/AKT/FASN cascade. Mol Carcinog 2019; 58: 31-41.

19. Hamy AS, Tury S, Wang X, et al. Celecoxib With Neoadjuvant Chemotherapy for Breast Cancer Might Worsen Outcomes Differentially by COX-2 Expression and ER Status: Exploratory Analysis of the REMAGUS02 Trial. J Clin Oncol 2019; 37: 624-35.

20. Tian J, Guo F, Chen Y, et al. Nanoliposomal formulation encapsulating celecoxib and genistein inhibiting COX-2 pathway and Glut-1 receptors to prevent prostate cancer cell proliferation. Cancer Letters 2019; 448: 1-10.

21. Han Y, Chen P, Zhang Y, et al.Synergy between Auranofin and Celecoxib against Colon Cancer In vitro and In vivo through a Novel Redox-Mediated Mechanism. Cancers. 2019; 11.

22. Yi L, Zhang $\mathrm{W}$, Zhang $\mathrm{H}$, et al. Systematic review and meta-analysis of the benefit of celecoxib in treating advanced non-small-cell lung cancer. Drug Des Devel Ther 2018; 12: 2455-66.

23. Jose $\mathrm{P}$, Sundar $\mathrm{K}$, Anjali $\mathrm{CH}$, et al. Metformin-loaded BSA nanoparticles in cancer therapy: a new perspective for an old antidiabetic drug. Cell Biochem Biophys 2015; 71: 627-36.

24. Ben Sahra I, Marchand-Brustel Y Le, Tanti JF, et al. Metformin in cancer therapy: a new perspective for an old antidiabetic drug? Mol Cancer Ther 2010; 9: 1092-99.

25. Chen $\mathrm{K}, \mathrm{Li} \mathrm{Y}, \mathrm{Guo} \mathrm{Z}$, et al. Metformin: current clinical applications in nondiabetic patients with cancer. Aging. 2020; 12

26. Marcucci F, Romeo E, Caserta CA, et al. Context-Dependent Pharmacological Effects of Metformin on the Immune System. Trends Pharmacol Sci 2020; 41: $162-71$
27. Lee J, Yesilkanal AE, Wynne JP, et al. Effective breast cancer combination therapy targeting BACH1 and mitochondrial metabolism. Nature. 2019; 568: 254-58.

28. Iranshahy M, Rezaee R AND Karimi G. Hepatoprotective activity of metformin: A new mission for an old drug? Eur J Pharmacol 2019; 850: 1-07.

29. Abdelmonsif DA, Sultan AS, EI-Hadidy WF, et al. Targeting AMPK, mTOR and $\beta$-Catenin by Combined Metformin and Aspirin Therapy in HCC: An Appraisal in Egyptian HCC Patients. Mol Diagn Ther 2018; 22: 115-27.

30. Faivre S, Rimassa L and Finn RS. Molecular therapies for HCC: Looking outside the box. J Hepatol 2020; 72: 342-52

31. Wang X, Chen X, Zhou H, et al. The Long Noncoding RNA, LINC01555, Promotes Invasion and Metastasis of Colorectal Cancer by Activating the Neuropeptide, Neuromedin U. Med Sci Monit 2019; 25: 4014-24.

32. Zhang $\mathrm{HH}$, Zhang $\mathrm{Y}$, Cheng $\mathrm{YN}$, et al. Metformin incombination with curcumin inhibits the growth, metastasis, and angiogenesis of hepatocellular carcinoma in vitro and in vivo. Mol Carcinog 2018; 57: 44-56.

33. Tabolaci C, Rossi S, Lentini A, et al. Aloin enhances cisplatin antineoplastic activity in B16-F10 melanoma cells by transglutaminase-induced differentiation. Amino acids 2013; 44: 293-300.

34. Cervello M, Bachvarov D, Lampiasi N, et al. Novel combination of sorafenib and celecoxib provides synergistic anti-proliferative and pro-apoptotic effects in human liver cancer cells. PLoS One 2013; 8: e65569.

35. Pritchard R, Rodríguez-Enríquez S, Pacheco-Velázquez SC, et al. Celecoxib inhibits mitochondrial $\mathrm{O}$ consumption, promoting ROS dependent death of murine and human metastatic cancer cells via the apoptotic signalling pathway. Biochem Pharmacol 2018; 154: 318-34.

36. Chu TH, Chan $\mathrm{HH}, \mathrm{Hu} \mathrm{TH}$, et al. Celecoxib enhances the therapeutic efficacy of epirubicin for Novikoff hepatoma in rats. Cancer Med 2018; 7: 2567-80.

37. Alamdarsaravi M, Ghajar A, Noorbala AA, et al. Efficacy and safety of celecoxib monotherapy for mild to moderate depression in patients with colorectal cancer: A randomized double-blind, placebo controlled trial. Psychiatry Res 2017; 255: 59-65.

38. Elzoghby AO, Mostafa SK, Helmy MA, et al. Multi-Reservoir Phospholipid Shell Encapsulating Protamine Nanocapsules for Co-Delivery of Letrozole and Celecoxib in Breast Cancer Therapy. Pharm Res 2017; 34: 1956-69.

39. Flamiatos JF, Beer TM, Graff JN, et al. Cyclooxygenase-2 (COX-2) inhibition for prostate cancer chemoprevention: double-blind randomised study of pre-prostatectomy celecoxib or placebo. BJU Int 2017; 119: 709-16.

40. Reckamp KL, Koczywas M, Cristea MC, et al. Randomized phase 2 trial of erlotinib in combination with high-dose celecoxib or placebo in patients with advanced non-small cell lung cancer. Cancer 2015; 121: 3298-306.

41. Toriyama S, Horinaka M, Yasuda S, et al. A Histone Deacetylase Inhibitor, OBP-801, and Celecoxib Synergistically Inhibit the Cell Growth with Apoptosis via a DR5-Dependent Pathway in Bladder Cancer Cells. Mol Cancer Ther 2016; 15: 2066-75.

42. Gao JH, Wen SL, Feng S, et al. Celecoxib and octreotide synergistically ameliorate portal hypertension via inhibition of angiogenesis in cirrhotic rats. Angiogenesis 2016; 19: 501-11.

43. Behr CA, Hesketh AJ, Barlow M, et al. Celecoxib inhibits Ewing sarcoma cell migration via actin modulation. J Surg Res 2015; 198: 424-33.

44. Steinbach G, Lynch PM, Phillips RK, et al. The effect of celecoxib, a cyclooxygenase-2 inhibitor, in familial adenomatous polyposis. N Engl J Med 2000; 342: 1946-52

45. Arrieta O, Barron F, Padilla MS, et al. Effect of Metformin Plus Tyrosine Kinase Inhibitors Compared With Tyrosine Kinase Inhibitors Alone in Patients With Epidermal Growth Factor Receptor-Mutated Lung Adenocarcinoma: A Phase 2 Randomized Clinical Trial. JAMA Oncol. 2019; p: e192553.

46. Au Yeung SL and Schooling CM. Impact of glycemic traits, type 2 diabetes and metformin use on breast and prostate cancer risk: a Mendelian randomization study. BMJ Open Diabetes Res Care 2019; 7: e872.

47. Vernieri C, Galli F, Ferrari L, et al. Impact of Metformin Use and Diabetic Status During Adjuvant Fluoropyrimidine-Oxaliplatin Chemotherapy on the Outcome of Patients with Resected Colon Cancer: A TOSCA Study Subanalysis. Oncologist 2019; 24: 385-93.

48. Tseng $\mathrm{CH}$. Metformin and risk of hepatocellular carcinoma in patients with type 2 diabetes. Liver Int 2018; 38: 2018-27.

49. Chen TW, Liang YN, Feng D, et al. Metformin inhibits proliferation and promotes apoptosis of HER2 positive breast cancer cells by downregulating HSP90. J BUON 2013; 18: 51-56.

50. Ko JC, Chiu HC, Wo TY, et al. Inhibition of p38 MAPK-dependent MutS homologue-2 (MSH2) expression by metformin enhances gefitinib-induced cytotoxicity in human squamous lung cancer cells. Lung Cancer 2013; 82: 397-406.

51. Erices R, Bravo ML, Gonzalez P et al. Metformin, at concentrations corresponding to the treatment of diabetes, potentiates the cytotoxic effects of carboplatin in cultures of ovarian cancer cells. Reprod Sci 2013; 20: 1433-46.

52. Liu J, Hou M, Yuan T et al. Enhanced cytotoxic effect of low doses of metformin combined with ionizing radiation on hepatoma cells via ATP deprivation and inhibition of DNA repair. Oncol Rep 2012; 28: 1406-12.

53. Dovedi SJ, Kirby JA, Davies BR, et al. Celecoxib has potent antitumour effects as a single agent and in combination with BCG immunotherapy in a model of urothelial cell carcinoma. Eur Urol 2008; 54: 621-30.

54. Gao J, Jia WD, Li JS, et al. Combined inhibitory effects of celecoxib and fluvastatin on the growth of human hepatocellular carcinoma xenografts in nude mice. J Int Med Res 2010; 38: 1413-27. 
55. Cervello M, Bachvarov D, Lampiasi N, et al. Novel combination of sorafenib and celecoxib provides synergistic anti-proliferative and pro-apoptotic effects in human liver cancer cells. PLoS One 2013; 8: e65569.

56. Ullah A, Ashraf M, Javeed A, et al. Enhancement of anti-proliferative activities of Metformin, when combined with Celecoxib, without increasing DNA damage. Environ Toxicol Pharmacol 2016; 45: 227-34.

57. Liu GY, Sabatini DM. mTOR at the nexus of nutrition, growth, ageing and disease. Nat Rev Mol Cell Biol. 2020;21:183-203.

58. Grabiner BC, Nardi V, Birsoy K, et al. A diverse array of cancer-associated MTOR mutations are hyperactivating and can predict rapamycin sensitivity. Cancer Discov 2014; 4: 554-63.

59. Ham G, Gong H, Wang Y, et al. AMPK/mTOR-mediated inhibition of survivin partly contributes to metformin-induced apoptosis in human gastric cancer cell. Cancer Biol Ther 2015; 16: 77-87.

60. Rocha GZ, Dias MM, Ropelle ER, et al. Metformin amplifies chemotherapy-induced AMPK activation and antitumoral growth. Clin Cancer Res 2011; 17: 3993-4005.

61. Strillacci A, Griffoni C, Valerii MC, et al. RNAi-based strategies for cyclooxygenase-2 inhibition in cancer. J Biomed Biotechnol 2010; 2010: 828045. 